\title{
Efficacy of Hydroxychloroquine in Patients with COVID-19 (Chen et al., 2020): Moderate Evidence for a Treatment Effect on Pneumonia
}

Eric-Jan Wagenmakers

Department of Psychological Methods

University of Amsterdam

Nieuwe Achtergracht 129 B

1018 WT Amsterdam, The Netherlands

E-mail: EJ.Wagenmakers@gmail.com

Tel.: +31645626624

Quentin F. Gronau

Department of Psychological Methods

University of Amsterdam

Nieuwe Achtergracht 129 B

1018 WT Amsterdam, The Netherlands

E-mail: Quentin.F.Gronau@gmail.com

Tel.: +49 1746977625

Abstract: A recent randomized clinical trial assessed the efficacy of hydroxychloroquine (HCQ) in the treatment of patients with common coronavirus disease-19 (COVID-19). ${ }^{1}$ The results showed that "the body temperature recovery time and the cough remission time were significantly shortened in the HCQ treatment group. Besides, a larger proportion of patients with improved pneumonia in the HCQ treatment group (80.6\%, 25 of 31) compared with the control group (54.8\%, 17 of 31)." A Bayesian reanalysis of the pneumonia data provides support for the hypothesis that HCQ improves pneumonia in patients with COVID-19. However, the degree of this support is moderate. In line with the authors' conclusion, more data are needed to draw definite conclusions. 
A recent randomized clinical trial assessed the efficacy of hydroxychloroquine (HCQ) in the treatment of patients with common coronavirus disease-19 (COVID-19). ${ }^{1}$ The results showed that "the body temperature recovery time and the cough remission time were significantly shortened in the HCQ treatment group. Besides, a larger proportion of patients with improved pneumonia in the HCQ treatment group (80.6\%, 25 of 31) compared with the control group $(54.8 \%, 17$ of 31$)$." The authors concluded: "Among patients with COVID-19, the use of HCQ could significantly shorten TTCR [time to clinical recovery] and promote the absorption of pneumonia".

This conclusion leaves unaddressed the degree to which the data undercut or support the hypothesis that $\mathrm{HCQ}$ is (in)effective. Here we focus on the data concerning pneumonia and quantify evidence by conducting a Bayesian logistic regression. ${ }^{2,3}$ Under the no-effect model $\mathrm{H}_{0}$, the log odds ratio equals $\psi=0$, whereas under the positive-effect model $\mathrm{H}_{+}, \psi$ is assigned a positive-only normal prior $\mathrm{N}_{+}(\mu, \sigma)$. A default analysis (i.e., $\mu=0, \sigma=1$ ) reveals moderate 4 evidence for $\mathrm{H}_{+}$: the data are 6 times more likely under the hypothesis that $\mathrm{HCQ}$ is beneficial than under the hypothesis that it is ineffective. Figure 1 shows the evidence is weak or moderate for all combinations of $\mu$ in $[0, .30]$ and $\sigma$ in $[0.1,1]$.

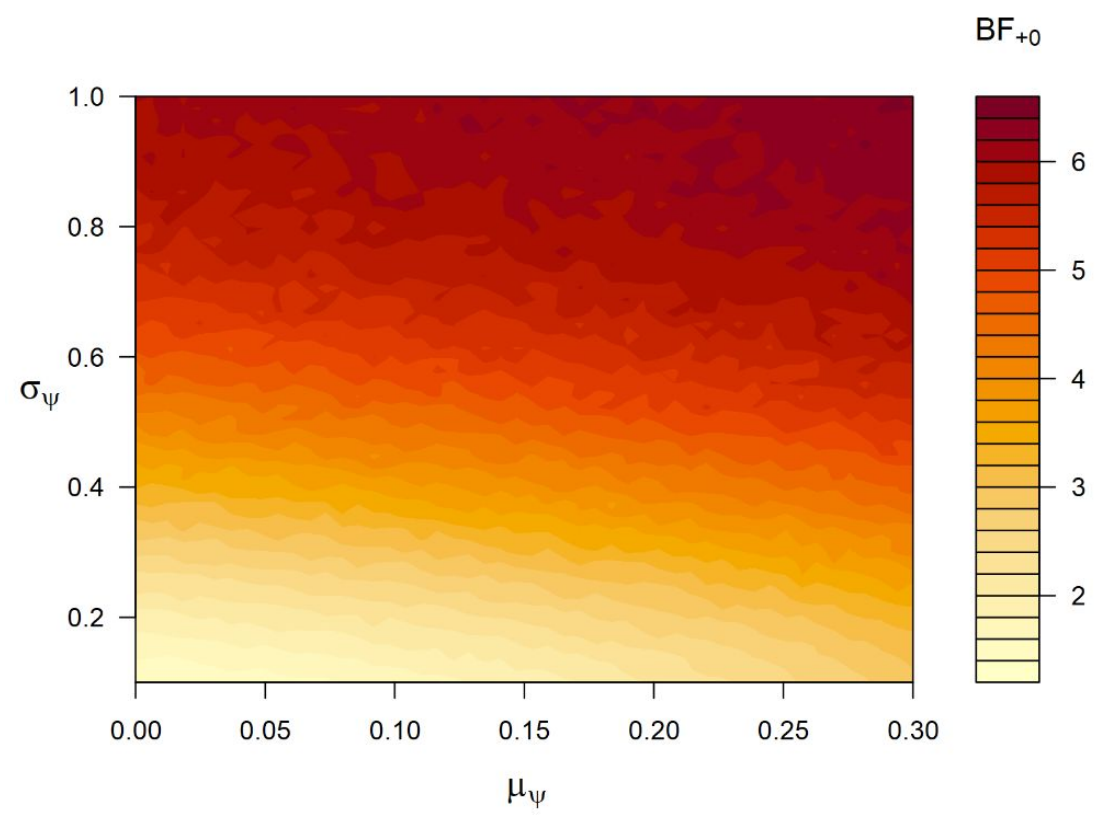

Figure 1. Across a range of different priors, the evidence for the positive-effect $H_{+}$over the no-effect $H_{0}$ is either weak or moderate.

In addition to hypothesis testing one may also inspect the posterior distribution for $\psi$ under a two-sided model that assigns $\psi$ a standard normal distribution. As Figure 2 shows, the posterior distribution is relatively wide, indicating that, under the assumption that the treatment is beneficial, there remains considerable uncertainty about the size of the benefit. 


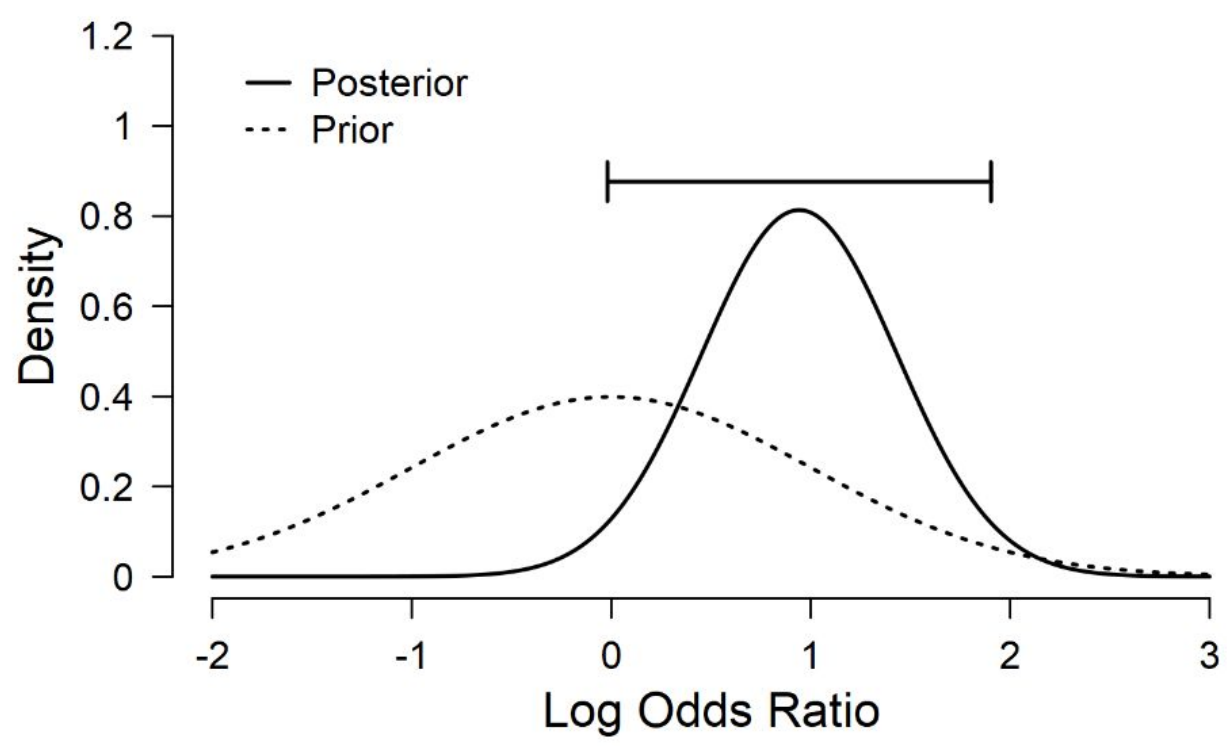

Figure 2. The posterior distribution for the log odds ratio is relatively wide, signifying a lack of certainty regarding the strength of the treatment effect (assuming it is present).

In sum, the data under consideration support the hypothesis that HCQ improves pneumonia in patients with COVID-19. However, the degree of this support is moderate. In line with the authors' conclusion, more data are needed to draw definite conclusions.

\section{References}

1. Chen, Z., Hu, J., Zhang, Z., Jiang, S., Han, S., Yan, D., Zhuang, R., Hu, B., \& Zhang, Z. (2020). Efficacy of hydroxychloroquine in patients with COVID-19: results of a randomized clinical trial.<LINK:

https://www.medrxiv.org/content/10.1101/2020.03.22.20040758v2>. Medrxiv.

2. Kass RE, Vaidyanathan SK. Approximate Bayes factors and orthogonal parameters, with application to testing equality of two binomial proportions. Journal of the Royal Statistical Society: Series B (Methodological) 1992;54:129-44.

3. Gronau QF, Raj K. N. A., Wagenmakers EJ. (2019). Informed Bayesian inference for the A/B test. Manuscript submitted for publication and available on arXiv: http://arxiv.org/abs/1905.02068

4. Jeffreys, H. Theory of Probability. 1st ed. Oxford University Press, Oxford, UK, 1939.

Funding: -

Conflict of Interest Statement: There are no conflicts of interest to report. 